\title{
DINÁMICA DE LAS EXPORTACIONES FRUTÍCOLAS CHILENAS DESDE UNA PERSPECTIVA REGIONAL (2008-2018)
}

\section{DYNAMICS OF CHILEAN FRUIT EXPORTS FROM A REGIONAL PERSPECTIVE (2008-2018)}

\author{
Sofía Boza ${ }^{1,2 *}$, Jazmín Muñoz ${ }^{1}$, Aracely Núñez ${ }^{2}$, y Jorge Díaz-Lanchas ${ }^{3}$ \\ ${ }^{1}$ Departamento de Economía Agraria, Facultad de Ciencias Agronómicas, Universidad de Chile, Santa \\ Rosa 11315, La Pintana, Santiago, Chile. \\ 2 Instituto de Estudios Internacionales, Universidad de Chile, Condell 249, Providencia, Santiago, Chile. \\ ${ }^{3}$ Universidad Loyola Andalucía, Campus Sevilla, Avenida de las Universidades, Dos Hermanas, \\ Sevilla, España. \\ * Autora para correspondencia E-mail: sofiaboza@u.uchile.cl.
}

\section{RESUMEN}

Las exportaciones de productos silvoagropecuarios, particularmente las exportaciones de fruta, son uno de los pilares de la economía chilena. En general, el estudio de este tipo de exportaciones en Chile se ha realizado desde una perspectiva nacional, a pesar de la gran diversidad de las condiciones agroclimáticas nacionales. El objetivo del presente artículo es caracterizar la evolución y dinámicas de las exportaciones frutícolas chilenas en la última década, pero desde una perspectiva regional. Para ello se utilizó información de fuentes oficiales, la cual se analizó mediante técnicas de estadística descriptiva y la construcción de diversos índices. Los resultados obtenidos muestran que las exportaciones de fruta se concentran en la zona central del país, donde las regiones tienen una mayor participación, así como también una importante oferta de productos frutícolas ofrecidos a diferentes mercados internacionales.

Palabras clave: exportaciones, frutas, fruticultura, regiones, Chile.

\begin{abstract}
Forest and agricultural exports, particularly fruit exports, are one of the backbones of the Chilean economy. In general, the study of this type of exports has been approached from a local perspective, regardless of the great diversity of agroclimatic conditions in the country. The objective of this article is to characterize the evolution and dynamics in of Chilean fruit exports the last decade from a regional perspective. For this, data were obtained from official sources and analyzed using descriptive statistics techniques and various indexes. The results obtained show that fruit is mainly exported from the central area of the country, where the regions have a greater share of the exports and also an important offer of fruits exported to different international markets.
\end{abstract}

Key words: exports, fruits, fruit production, regions, Chile.

Recibido: 08 enero $2020 . \quad$ Aceptado: 29 enero 2020. 


\section{INTRODUCCIÓN}

Chile dedica gran parte de su producción agrícola a la exportación. Según el Banco Central (2019) el país registró un valor de las exportaciones de 75.482 miles de millones de dólares en 2018; aproximadamente un cuarto de ello corresponde a productos silvoagropecuarios (SAP). De hecho, la balanza comercial SAP de Chile ha presentado una tendencia al crecimiento continuado desde 1990 a 2018 del 8,6\%. No obstante, no todos los rubros ni todos los mercados de destino tienen la misma participación en las exportaciones. Estados Unidos, China y la Unión Europea acumulan casi el 55\% del valor de las exportaciones SAP chilenas según datos de 2018. Uno de los principales rubros que participa del comercio exterior SAP chileno son las frutas, y sobre todo algunas especies como las uvas de mesa, las manzanas y los berries. Chile es en la actualidad de hecho el sexto exportador mundial de frutas, y líder en el hemisferio sur.

La importancia de las exportaciones frutícolas ha conllevado un amplio interés en su análisis; principalmente desde una perspectiva nacional (López-Campos et al., 2019; Losilla et al., 2019). Sin embargo, ha sido mucho más escaso su estudio de manera desagregada a partir de las dinámicas de unidades territoriales más específicas como son las regiones; y los existentes se han centrado en ciertos productos y zonas concretas (Almonacid, 2018; Jara-Rojas et al., 2018). En Chile, donde se reconoce la existencia de una alta variedad de condiciones edafoclimáticas para la producción agrícola (Sarricolea et al., 2017) esta reflexión se hace aún más atingente. De hecho, dichas condiciones son un factor objetivo que influye en el desarrollo agrícola relativo de las regiones. En efecto, la zona central y centro sur concentra gran parte de la producción frutícola de exportación, en términos tanto de volumen como de valor. Sin embargo, hay otros elementos que potencialmente influyen en el perfil exportador de las regiones, como son la infraestructura y la capacidad logística (Bensassi, et al, 2015).

La inserción en los mercados de exportación supone una oportunidad, pero también deriva en una brecha, entre productores. En Chile los agricultores orientados al mercado nacional tienen un notorio atraso en uso de tecnologías, acceso a financiamiento e incluso a programas públicos comparados con aquellos focalizados en la exportación (Echeverría et al., 2012). Ríos y Torres (2014) afirmaron que la agricultura chilena se caracteriza por una marcada dualidad entre una minoría de empresas medianas y grandes exportadoras y una mayoría de micro y pequeños productores orientados al mercado local, con significativos contrastes. En consecuencia, el perfil exportador de las regiones también puede orientar respecto a la situación de sus productores.

El objetivo del presente artículo es, por tanto, caracterizar la evolución y dinámicas en la última década de las exportaciones frutícolas chilenas, pero desde una perspectiva regional, abordando de manera desagregada la totalidad del territorio y también los distintos productos.

\section{MATERIALES Y MÉTODOS}

La información para la realización del presente artículo se obtuvo de las bases de datos sobre exportaciones agrícolas regionales de la Oficina de Estudios y Políticas Agrarias (ODEPA, 2019) entre los años 2008 y 2018. En ellas se tiene clasificadas por códigos del Sistema Armonizado (SA) información detallada respecto tanto a valores como a volúmenes de exportación, por mercados de destino. Respecto de dichos datos, se realizó en primer lugar un análisis de tipo descriptivo, que se centró en la participación actual y evolución de las distintas regiones en las exportaciones frutícolas nacionales, así como sus principales mercados de destino y productos.

Éste se complementó con el cálculo de dos índices: la Tasa de Crecimiento Anual Compuesto (TCAC) y el Índice de Herfindahl-Hirschmann (IHH). Respecto del primero, se trata de un indicador más propio del campo de las finanzas, pero también adaptable en este caso dado que calcula una tasa de crecimiento uniforme durante el periodo de tiempo considerado, ignorando la volatilidad de las exportaciones interanuales para efectos de simplificación (Sunny y Waheeda, 2010; Sufaira, 2016). Se considera el cociente del valor final e inicial del periodo, elevado a la inversa del número de años, cuyo resultado es restado de la unidad (transformable en porcentaje). Por su parte, el IHH es un indicador del grado de concentración en un determinado mercado. Uníndice mayor a 0,18 representa una canasta exportadora "concentrada", entre 0,10 y 0,18 "moderadamente concentrada", menor a 0,10 se considera "diversificada" (Durán y Álvarez, 2008). Este indicador se cruzó mediante un análisis de correlación, específicamente utilizando el coeficiente de Pearson, con la participación exportadora de las distintas regiones. Ello con el fin de poder tener una aproximación a la relación potencial entre la inserción en los mercados frutícolas internacionales y la concentración en términos tanto de destinos como de productos.

\section{RESULTADOS Y DISCUSIÓN}

Las exportaciones frutícolas de Chile presentan una tendencia creciente en el periodo 2008-2018, registrando un total de 3.939 millones USD en 


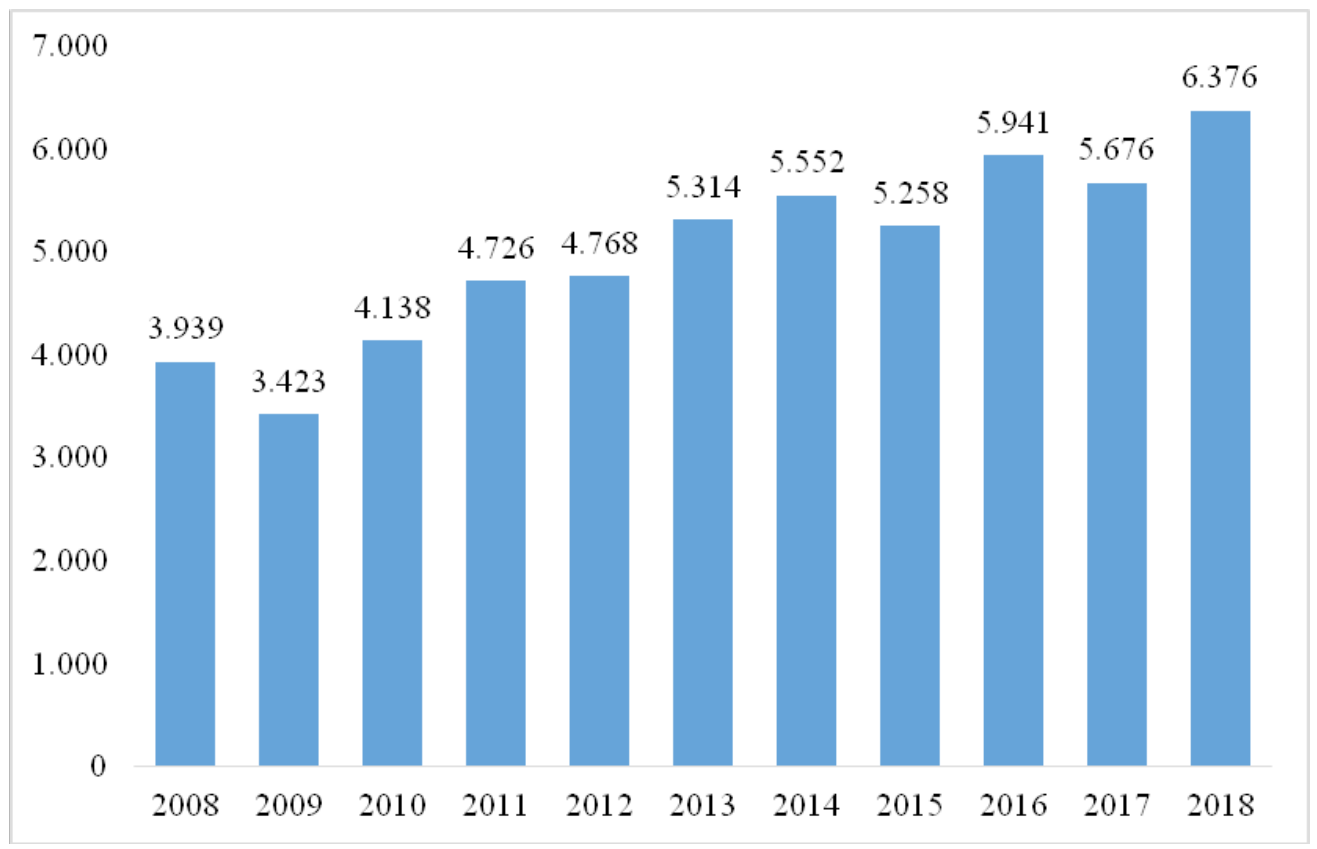

Fig. 1. Exportaciones frutícolas de Chile (millones USD), 2008-2018.

Fig. 1. Chilean fruit exports (million USD), 2008-2018.

2008 a 6.376 millones USD en 2018 con una TCAC del $5 \%$.

La Región de O’Higgins permanece como la principal región exportadora frutícola de Chile en el periodo 2008-2018. En 2008 tuvo una participación del $26,7 \%$ de las exportaciones de fruta a nivel nacional, y el 33,47\% en 2018 . Las regiones de El Maule y Metropolitana, han aumentado su participación en el total de las exportaciones de fruta pasando del $15,89 \%$ al $17,66 \%$ y del $10,64 \%$ al $15,10 \%$, respectivamente, ocupando el segundo y tercer lugar. El cambio de posición de dichas regiones se relaciona con la caída de Valparaíso del segundo lugar $(23,72 \%)$ en 2008, al cuarto lugar (14,97\%) en 2018. Por su parte, Coquimbo mantiene el quinto lugar en $2008(9,53 \%)$ y $2018(8,42 \%)$. El resto de las regiones presenta una variación poco significativa en la participación del total de exportaciones y el cambio de posiciones es débil (Tabla 1).

La evolución de las exportaciones de frutas es muy distinta entre las regiones (Fig. 2, Tabla 2). Muestra de ello, son los distintos resultados obtenidos al calcular la TCAC para cada región tomando como año inicial el 2008 y año final 2018. Así se evidencia que, aunque la mayoría de las regiones experimentaron un crecimiento durante esos años (excepto Atacama, Tarapacá, Arica y Parinacota) se obtuvieron TCAC desde 0,2\% (Valparaíso) y hasta 70,5\% (Los Ríos). De tal forma que la Región de Los Ríos registra exportaciones de alrededor de 0,09 millones USD en 2008 y cerca de 18,73 millones USD en 2018, lo cual representa una TCAC del 70,5\%. Aunque la Región de O'Higgins presenta una TCAC moderada $(7,3 \%)$, durante casi todo el periodo lidera las exportaciones frutícolas de Chile (excepto en 2009, ligeramente superada por Valparaíso).

La participación regional del total nacional de exportaciones frutícolas, así como la principal fruta de exportación por cada región se presenta en la Fig. 3. En todas las regiones el principal producto que se exporta tiene una participación que supera al menos el $25 \%$ del valor total. No obstante, en aquellas con baja participación en las exportaciones frutícolas nacionales, el peso del principal producto exportado es aún mayor (en algunas más del 90\%). En específico, en la Región de $\mathrm{O}^{\prime}$ Higgins el principal producto exportado son carozos y cerezas (41\%); en El Maule son las manzanas y peras (31\%); en la Región Metropolitana las nueces $(26 \%)$ y en Valparaíso y Coquimbo las uvas (14,97 y 8,42\%, respectivamente).

En concordancia con lo anteriormente descrito, en el Tabla 3 se presentan los resultados del cálculo del Índice Herfindahl-Hirschmann (IHH) respecto a la diversificación/concentración en productos, entre los años 2008-2018. Se obtuvo que casi todas las regiones presentan una alta concentración en pocas frutas de exportación: 
Tabla 1. Participación de exportaciones frutícolas de Chile, por regiones, 2008 y 2018.

Table 1. Region's share of Chilean fruit exports, 2008 and 2018.

\begin{tabular}{|c|c|c|c|c|c|}
\hline \multirow{2}{*}{$\begin{array}{l}\text { Región } \\
\text { O'Higgins }\end{array}$} & \multicolumn{2}{|c|}{$\begin{array}{l}\text { Participación del } \\
\text { total de } \\
\text { exportaciones } 2008 \\
\text { y Ranking } \\
\end{array}$} & \multicolumn{2}{|c|}{$\begin{array}{l}\text { Participación del } \\
\text { total de exportaciones } \\
2018 \text { y Ranking }\end{array}$} & \multirow[t]{2}{*}{ 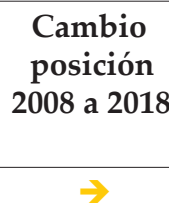 } \\
\hline & $26,70 \%$ & $1^{\circ}$ & $33,47 \%$ & $1^{\circ}$ & \\
\hline Maule & $15,89 \%$ & $3^{\circ}$ & $17,66 \%$ & $2^{\circ}$ & $\uparrow$ \\
\hline Metropolitana & $10,64 \%$ & $4^{\circ}$ & $15,10 \%$ & $3^{\circ}$ & $\uparrow$ \\
\hline Valparaíso & $23,72 \%$ & $2^{\circ}$ & $14,97 \%$ & $4^{\circ}$ & $\downarrow$ \\
\hline Coquimbo & $9,53 \%$ & $5^{\circ}$ & $8,42 \%$ & $5^{\circ}$ & $\Rightarrow$ \\
\hline Biobío $^{1}$ & $4,93 \%$ & $7^{\circ}$ & $4,50 \%$ & $6^{\circ}$ & $\uparrow$ \\
\hline Atacama & $6,77 \%$ & $6^{\circ}$ & $3,07 \%$ & $7^{\circ}$ & $\downarrow$ \\
\hline La Araucanía & $0,90 \%$ & $8^{\circ}$ & $1,97 \%$ & $8^{\circ}$ & $\Rightarrow$ \\
\hline Los Lagos & $0,71 \%$ & $9^{\circ}$ & $0,45 \%$ & $9^{\circ}$ & $\Rightarrow$ \\
\hline Los Ríos & $0,00 \%$ & $14^{\circ}$ & $0,29 \%$ & $10^{\circ}$ & $\uparrow$ \\
\hline Antofagasta & $0,03 \%$ & $11^{\circ}$ & $0,04 \%$ & $11^{\circ}$ & $\Rightarrow$ \\
\hline Gral. C. I. del Campo & $0,01 \%$ & $13^{\circ}$ & $0,02 \%$ & $12^{\circ}$ & $\downarrow$ \\
\hline Tarapacá & $0,02 \%$ & $12^{\circ}$ & $0,01 \%$ & $13^{\circ}$ & $\uparrow$ \\
\hline Arica y Parinacota & $0,15 \%$ & $10^{\circ}$ & $0,00 \%$ & $14^{\circ}$ & $\downarrow$ \\
\hline Magallanes & $0,00 \%$ & $15^{\circ}$ & $0,00 \%$ & $15^{\circ}$ & $\Rightarrow$ \\
\hline
\end{tabular}

${ }^{1}$ Incluye la Región de Nuble, que existe como tal desde 2018.

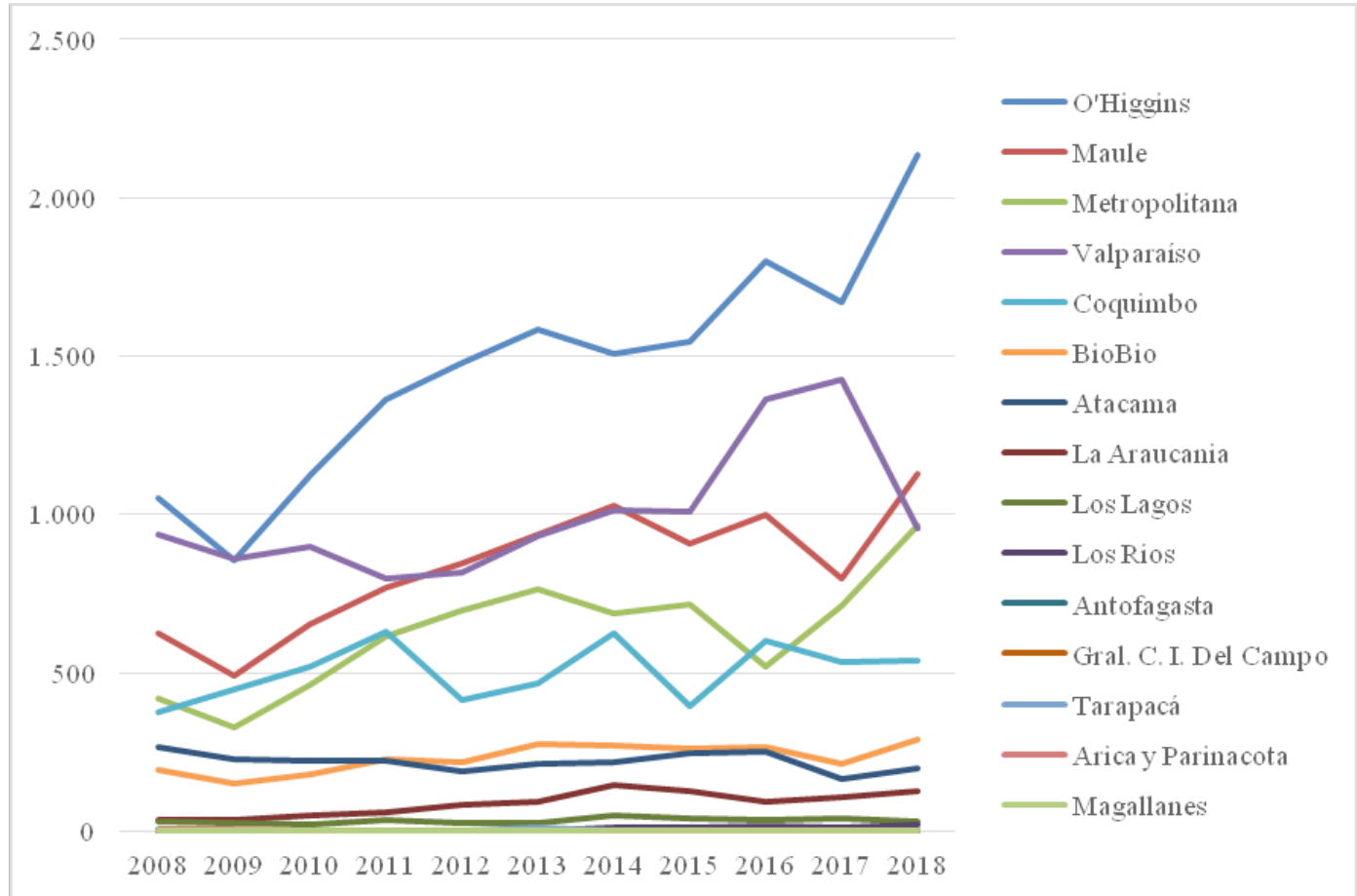

Fig. 2. Evolución exportaciones de fruta (millones USD FOB), por regiones, 2008-2018.

Fig. 2. Evolution of fruit exports (million USD FOB) by region, 2008-2018. 
Tabla 2. Exportación de frutas (Millones de USD FOB), TCAC 2008-2018, por regiones. Table 2. Fruit exports (Millions of USD FOB) by region, CAGR 2008-2018.

\begin{tabular}{lrrr}
\hline & $\mathbf{2 0 0 8}$ & $\mathbf{2 0 1 8}$ & TCAC 2008-2018 \\
\hline O’Higgins & $1.051,56$ & $2.134,37$ & $7,3 \%$ \\
Maule & 625,83 & $1.126,09$ & $6,1 \%$ \\
Metropolitana & 419,07 & 962,91 & $8,7 \%$ \\
Valparaíso & 934,15 & 954,73 & $0,2 \%$ \\
Coquimbo & 375,25 & 537,18 & $3,7 \%$ \\
BioBio & 194,15 & 286,86 & $4,0 \%$ \\
Atacama & 266,49 & 196,06 & $-3,0 \%$ \\
La Araucanía & 35,42 & 125,92 & $13,5 \%$ \\
Los Lagos & 28,04 & 28,95 & $0,3 \%$ \\
Los Ríos & 0,09 & 18,73 & $70,1 \%$ \\
Antofagasta & 1,34 & 2,54 & $6,6 \%$ \\
Gral. C. I. del Campo & 0,50 & 1,47 & $11,3 \%$ \\
Tarapacá & 0,96 & 0,47 & $-6,8 \%$ \\
Arica y Parinacota & 5,87 & 0,07 & $-36,1 \%$ \\
Magallanes & 0,02 & 0,03 & $6,1 \%$ \\
\hline
\end{tabular}

TCAC: Tasa de Crecimiento Anual Compuesto

11 de las 15 regiones analizadas tienen un $\mathrm{IHH}$ producto promedio superior a 0,18 . Por su parte, los resultados del IHH producto promedio de las regiones de Antofagasta, La Araucanía, Maule y Metropolitana indican moderada concentración. En general, no se observan cambios considerables en los patrones de concentración durante el periodo analizado, a excepción de Antofagasta.

Dado que las regiones del Maule y Metropolitana presentan una moderada concentración de producto, y a su vez cuentan con una alta participación en las exportaciones nacionales de fruta, se realizó un análisis de correlación entre el IHH producto y las respectivas participaciones regionales. Obteniéndose un coeficiente de correlación de Pearson de $-0,3518$ estadísticamente significativo (p-valor 0,000), lo cual implica una relación inversa entre las variables analizadas; es decir, a mayor participación regional en las exportaciones nacionales de fruta, menor concentración de producto, y viceversa.

Respecto a los destinos de exportación de cada región (Fig. 4), se evidencia que el más común entre las regiones es Estados Unidos, el mismo que en 10 de 15 regiones se posiciona como principal destino de fruta. Cabe destacar el reciente protagonismo de China, al posicionarse en muchas regiones como primero o segundo mercado de exportación de fruta.

Respecto a la concentración de los destinos de exportación por regiones, los resultados del IHH destino (Tabla 4) muestran que existe diferentes niveles de concentración entre las regiones. La Región Metropolitana es la única que ha diversificado sus mercados de exportación durante todo el periodo analizado, con un $\mathrm{IHH}$ promedio de 0,07. Las regiones O'Higgins, Maule, Tarapacá y Valparaíso presentan una moderada concentración de sus destinos de exportación. Las regiones restantes tienen poca diversificación de sus mercados de exportación. Al igual que en el caso del IHH producto, se realizó un análisis de correlación entre el $\mathrm{IHH}$ destino y las respectivas participaciones regionales. Se obtuvo un coeficiente de correlación de Pearson de -0,4241 estadísticamente significativo ( $\mathrm{p}$-valor $0,000)$, lo cual implica una relación inversa entre las variables analizadas; a mayor participación regional en las exportaciones nacionales de fruta, menor concentración de mercados, y viceversa.

\section{CONCLUSIONES}

El presente artículo permite concluir que en Chile la exportación frutícola se encuentra concentrada principalmente en la zona central del país, ya que de las regiones entre Valparaíso y El Maule deriva el $81,2 \%$ de las mismas. De hecho, esta concentración se ha intensificado en la última década, sobre todo si nos enfocamos en las regiones de O'Higgins, Metropolitana y El Maule. El resto de las regiones del país han tenido y tienen una participación muy limitada.

Con relación a los productos comercializados, se observa que el principal de cada región concentra al menos un $25 \%$ del valor total de 


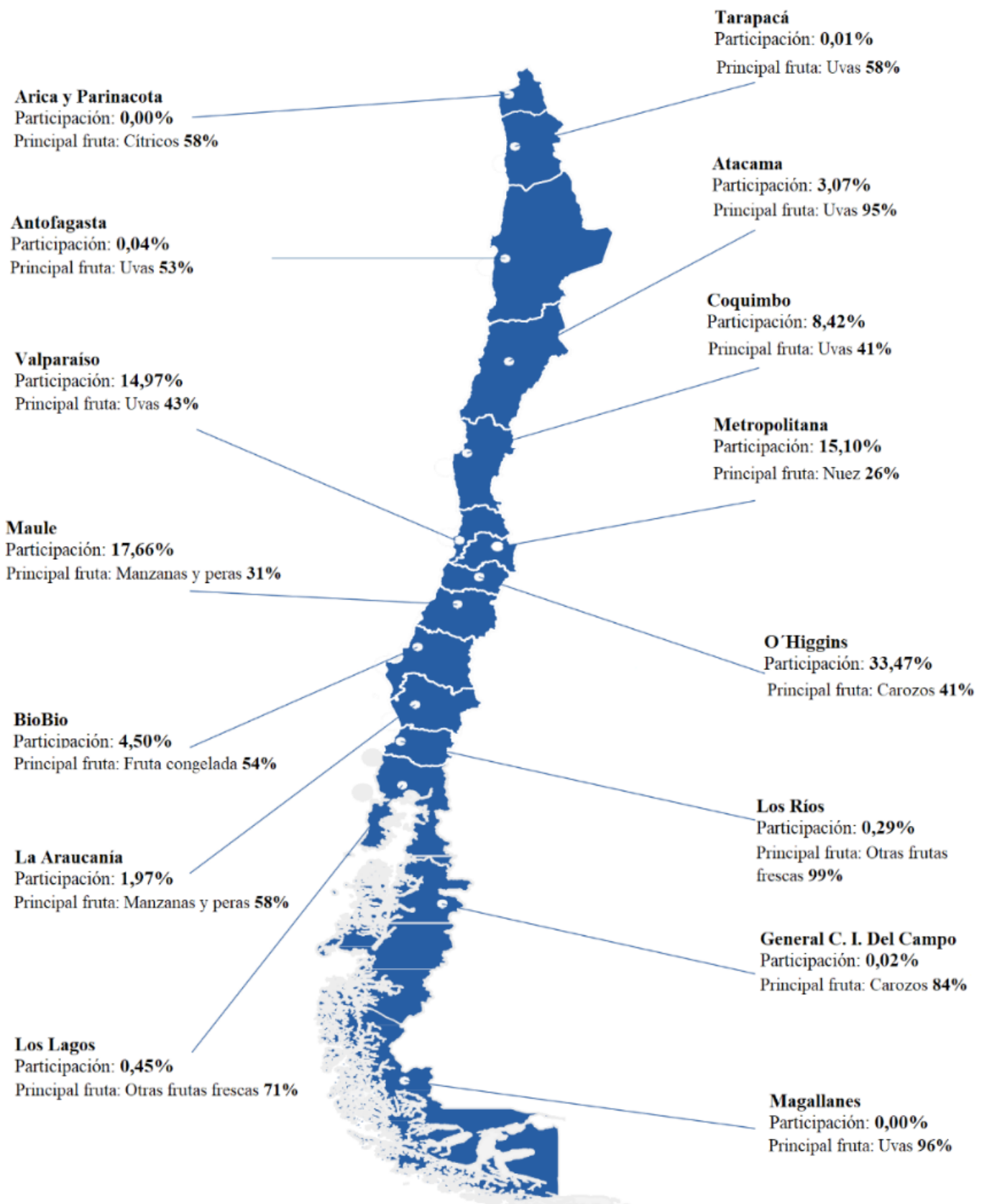

Fig. 3. Participación regional del total nacional de exportaciones frutícolas y fruta principal, 2018. Fig. 3. Region's share of total fruit exports and main exported fruit, 2018. 
Tabla 3. Índice Herfindahl-Hirschman ( $\mathrm{IHH}^{1}$ ) calculado según cantidad de productos exportados por región.

Table 3. Herfindahl-Hirschman Index (HHI) calculated based on the number of products exported by region.

\begin{tabular}{lcccccccccccc}
\hline & $\mathbf{2 0 0 8}$ & $\mathbf{2 0 0 9}$ & $\mathbf{2 0 1 0}$ & $\mathbf{2 0 1 1}$ & $\mathbf{2 0 1 2}$ & $\mathbf{2 0 1 3}$ & $\mathbf{2 0 1 4}$ & $\mathbf{2 0 1 5}$ & $\mathbf{2 0 1 6}$ & $\mathbf{2 0 1 7}$ & $\mathbf{2 0 1 8}$ & Promedio \\
\hline Antofagasta & 0,21 & 0,15 & 0,01 & 0,11 & 0,20 & 0,05 & 0,07 & 0,31 & 0,05 & 0,08 & 0,21 & 0,13 \\
Arica y Parinacota & 0,29 & 0,21 & 0,17 & 0,40 & 0,19 & 0,24 & 0,24 & 0,22 & 0,37 & 0,99 & 0,03 & 0,30 \\
Atacama & 0,92 & 0,85 & 0,88 & 0,87 & 0,83 & 0,90 & 0,87 & 0,84 & 0,77 & 0,84 & 0,87 & 0,86 \\
Biobío & 0,32 & 0,45 & 0,30 & 0,35 & 0,31 & 0,32 & 0,30 & 0,32 & 0,40 & 0,42 & 0,30 & 0,34 \\
Coquimbo & 0,46 & 0,39 & 0,41 & 0,44 & 0,43 & 0,43 & 0,38 & 0,29 & 0,26 & 0,18 & 0,23 & 0,35 \\
General C.I. Del & & & & & & & & & & & & \\
Campo & 0,09 & 0,28 & 0,31 & & & 0,22 & 0,69 & & & 0,23 & 0,58 & 0,34 \\
La Araucanía & 0,39 & 0,39 & 0,38 & 0,35 & 0,39 & 0,42 & 0,41 & 0,31 & 0,39 & 0,45 & 0,35 & 0,38 \\
O'Higgins & 0,17 & 0,16 & 0,14 & 0,16 & 0,16 & 0,16 & 0,16 & 0,14 & 0,17 & 0,12 & 0,17 & 0,16 \\
Los Lagos & 0,66 & 0,49 & 0,53 & 0,43 & 0,26 & 0,22 & 0,47 & 0,35 & 0,37 & 0,47 & 0,50 & 0,43 \\
Magallanes & 0,08 & 0,10 & 0,06 & 0,31 & 0,22 & 0,42 & 0,18 & 0,13 & 0,16 & 0,59 & 0,83 & 0,28 \\
Maule & 0,20 & 0,20 & 0,19 & 0,18 & 0,17 & 0,17 & 0,15 & 0,12 & 0,16 & 0,17 & 0,15 & 0,17 \\
Metropolitana & 0,13 & 0,13 & 0,12 & 0,12 & 0,15 & 0,18 & 0,16 & 0,18 & 0,16 & 0,17 & 0,11 & 0,15 \\
Los Ríos & & & 0,82 & 0,27 & 0,24 & 0,85 & 0,83 & 0,73 & 0,97 & 0,93 & 0,96 & 0,73 \\
Tarapacá & 0,14 & 0,31 & 0,24 & 0,23 & 0,23 & 0,66 & 0,07 & 0,10 & 0,26 & 0,14 & 0,24 & 0,24 \\
Valparaíso & 0,25 & 0,25 & 0,25 & 0,28 & 0,38 & 0,28 & 0,26 & 0,21 & 0,14 & 0,13 & 0,19 & 0,24 \\
\hline
\end{tabular}

${ }^{1}$ Concentración alta: rojo; concentración moderada: naranja; diversificación: verde.

Tabla 4. Índice Herfindahl-Hirschman ( $\left.\mathrm{IHH}^{1}\right)$ calculado según cantidad de países de destino, por región

Table 4. Herfindahl-Hirschman Index (IHH) calculated based on the number of export destinations by region

\begin{tabular}{l|cccccccccccc}
\hline & 2008 & 2009 & 2010 & 2011 & 2012 & 2013 & 2014 & 2015 & 2016 & 2017 & 2018 & Promedio \\
\hline $\begin{array}{l}\text { Antofagasta } \\
\text { Arica y }\end{array}$ & 0,46 & 0,08 & 0,19 & 0,15 & 0,36 & 0,42 & 0,12 & 0,13 & 0,06 & 0,05 & 0,33 & 0,22 \\
$\begin{array}{l}\text { Parinacota } \\
\text { Atacama }\end{array}$ & 0,22 & 0,29 & 0,20 & 0,01 & 0,22 & 0,24 & 0,80 & & 0,10 & & 0,03 & 0,23 \\
Biobío & 0,52 & 0,53 & 0,48 & 0,33 & 0,29 & 0,33 & 0,31 & 0,31 & 0,37 & 0,25 & 0,29 & 0,36 \\
Coquimbo & 0,22 & 0,21 & 0,24 & 0,27 & 0,26 & 0,23 & 0,16 & 0,16 & 0,16 & 0,14 & 0,14 & 0,20 \\
General C.I. & 0,39 & 0,40 & 0,31 & 0,28 & 0,30 & 0,28 & 0,29 & 0,30 & 0,24 & 0,32 & 0,31 \\
Del Campo & 0,09 & 0,07 & 0,31 & 0,47 & & & 0,69 & 0,73 & 0,48 & 0,50 & 0,24 & 0,40 \\
La Araucanía & 0,13 & 0,19 & 0,17 & 0,15 & 0,18 & 0,15 & 0,36 & 0,30 & 0,20 & 0,19 & 0,17 & 0,20 \\
O'Higgins & 0,12 & 0,12 & 0,12 & 0,09 & 0,09 & 0,10 & 0,12 & 0,14 & 0,16 & 0,13 & 0,17 & 0,12 \\
Los Lagos & 0,46 & 0,28 & 0,24 & 0,22 & 0,16 & 0,20 & 0,37 & 0,20 & 0,22 & 0,20 & 0,16 & 0,25 \\
Magallanes & & 0,97 & 0,98 & & 0,05 & 0,25 & 0,14 & 0,07 & 0,52 & 0,59 & 0,83 & 0,49 \\
Maule & 0,10 & 0,11 & 0,11 & 0,11 & 0,11 & 0,11 & 0,11 & 0,11 & 0,13 & 0,09 & 0,11 & 0,11 \\
Metropolitana & 0,07 & 0,09 & 0,07 & 0,08 & 0,08 & 0,09 & 0,05 & 0,05 & 0,05 & 0,05 & 0,08 & 0,07 \\
Los Ríos & 0,07 & 0,56 & 0,35 & 0,37 & 0,08 & 0,27 & 0,40 & 0,25 & 0,36 & 0,26 & 0,27 & 0,30 \\
Tarapacá & 0,11 & 0,25 & 0,18 & 0,09 & 0,25 & 0,24 & 0,06 & 0,08 & 0,18 & 0,15 & 0,02 & 0,15 \\
Valparaíso & 0,16 & 0,21 & 0,18 & 0,17 & 0,17 & 0,13 & 0,14 & 0,14 & 0,19 & 0,16 & 0,13 & 0,16 \\
\hline
\end{tabular}

${ }^{1}$ Concentración alta: rojo; concentración moderada: naranja; diversificación: verde. 


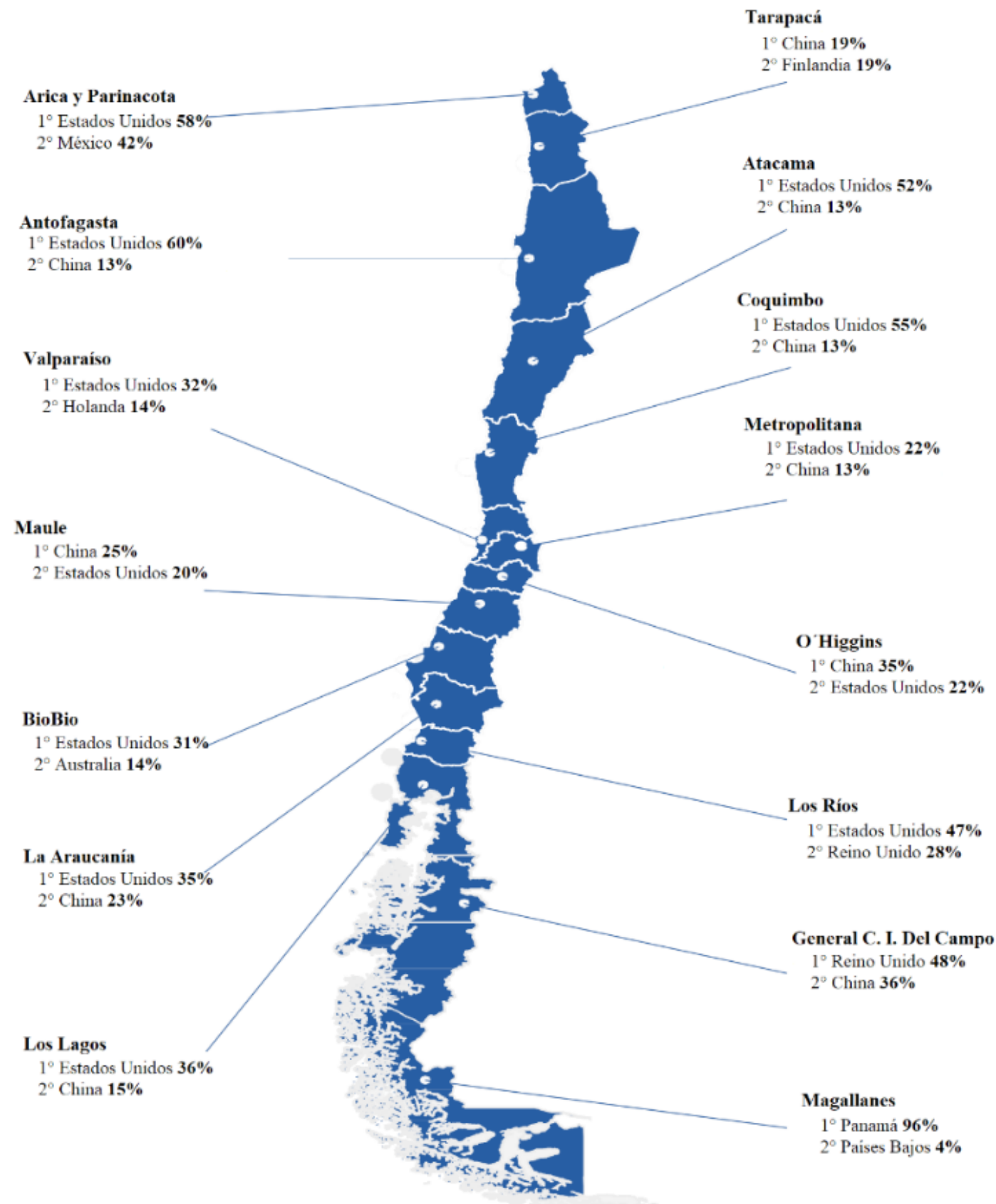

Fig. 4. Principales destinos de exportación de fruta, por regiones, 2018.

Fig. 4. Main destinations of fruit exports by region, 2018. 
las exportaciones. Respecto a los mercados de destino, en general, Estados Unidos ha estado posicionado tradicionalmente como el más relevante; no obstante, China se ha instalado de manera progresiva.

Los análisis realizados permiten, al menos $a$ priori, demostrar una cierta relación inversa entre la participación en las exportaciones frutícolas y la concentración de las mismas, tanto en productos como en mercados de destino. Lo anterior puede deberse a que se utilizan estrategias más complejas cuando la inserción en los mercados internacionales es mayor.

Los resultados en el presente artículo permiten una primera aproximación a la realidad de las exportaciones de un rubro tan relevante como el frutícola desde una perspectiva desagregada, lo que amplía el diagnóstico y facilita la toma de decisiones. Así mismo, se genera un espacio para poder investigar más en profundidad las diversas causas de los perfiles exportadores.

\section{RECONOCIMIENTOS}

Esta investigación fue desarrollada dentro del Programa de Estímulo a la Excelencia Institucional (PEEI) 2016-2017 financiado por la Universidad de Chile a través del Instituto de Estudios Internacionales de la misma Casa de Estudios.

\section{LITERATURA CITADA}

Almonacid, F. 2018. El sur de Chile como parte de cadenas globales de valor, 19852016: economía regional y producción de arándanos. Ager 25:131-158. doi:10.4422/ ager.2018.08

Banco Central. 2019. Balanza comercial de productos silvoagropecuarios Avance mensual enero-febrero 2019. ODEPA, Santiago, Chile. Disponible en https://www. odepa.gob.cl/wp-content/uploads/2019/02/ Balanza_febrero.pdf. (Consulta 6 enero 2020)

Bensassi, S., L. Márquez-Ramos, I. MartínezZarzoso, y C. Suárez-Burguet. 2015. Relationship between logistics infrastructure and trade: evidence from Spanish regional exports. Transportation Research Part A: Policy and Practice 72:47-61. doi:10.1016/j. tra.2014.11.007

Durán, J., M. Álvarez. 2008. Indicadores de comercio exterior y política comercial: mediciones de posición y dinamismo comercial, Santiago, Chile. Disponible en https://repositorio.cepal.org/bitstream/ handle/11362/3690/S2008794_es.pdf. (Consulta 6 enero 2020)
Echeverría, R., V. Moreira, J. Barrena, y M. Gopinath. 2012. A characterization of Chilean farmers based on their market-production orientation. Ciencia e Investigación Agraria 39:255-264.

Jara-Rojas, R., B. Bravo-Ureta, D. Solís, y D. Arriagada. 2018. Technical efficiency and marketing channels among small-scale farmers: Evidence for raspberry production in Chile. International Food and Agribusiness Management Review 21(3):351-364. doi:10.22434/IFAMR2016.0168

López-Campos, M., R. González-Ramírez, L. Bearzotti, y S. Cannella. 2018. Modelling and analysis of the apples export supply chain business processes: Experiences from Chile. p. 29-52. En J. García, L. Rivera, R. GonzálezRamírez, and G. Leal (eds.) Best Practices in Manufacturing Processes: Experiences from Latin America. Springer, Switzerland.

Losilla, L., A. Engler, y V. Otter. 2019. Internationalization paths of fruit export companies from emerging economies: Are they regionally or globally oriented? International Journal of Emerging Markets. doi: 10.1108/IJOEM-12-2017-0562

ODEPA. 2019. Oficina de Estudios y Políticas Agrarias. Bases de datos comercio exterior, Santiago, Chile. Disponible en https://www. odepa.gob.cl/estadisticas-del-sector/basesde-datos-comercio-exterior. (Consulta 6 enero 2020)

Ríos, S., y G. Torres. 2014. El sector agropecuario en la región de Los Lagos y el paradigma “Chile potencia alimentaria": desafíos para la política agraria nacional. Mundo Agrario 15(29).

Sarricolea, P., M. Herrera-Ossandon, y Ó. Meseguer-Ruíz. 2017. Climatic regionalisation of continental Chile. Journal of Maps 13(2):66-73. doi: 10.1080/17445647.2016.1259592

Sufaira, C. 2016. Growth and diversification of manufacturing export in India Since 1991 ProQuest. Journal of International Economics 1:111-123.

Sunny, T., y S. Waheeda. 2010. Growth and composition of Indian agricultural exports during reform era. National Monthly Refereed Journal of Research in Commerce \& Management 1(6):92-104. 